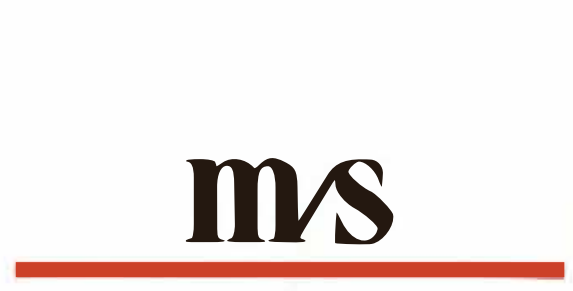

médecine/sciences $1994 ; 10: 1077-8$

\section{André Holley}

\section{RÉFÉRENCES}

1. Buck L, Axel R. A novel multigene family may encode odorant receptors: a molecular basis for odor recognition. Cell 1991 ; 65 : 175-87.

2. Michel D, Moyse E, Brun G, Jourdan F. Induction of apoptosis in rat olfactory neuroepithelium by synaptic target ablation. NeuroReport $1994 ; 5: 1329-32$.

3. Pellier V, Astic L. Detection of apoptosis by electron microscopy and in situ labelling, in the rat olfactory pit. NeuroReport $1994 ; 5$ : 1429-32.

4. Devon R, Doucette R. Olfactory ensheating cells myelinate dorsal root ganglion neurites. Brain Res 1992 ; 589 : 175-9.

5. Ramon-Cuelo A, Nieto-Sampedro M. Regeneration into the spinal cord of transfected dorsal root axons is promoted by ensheating glia transplants. Exp Neurol 1994 ; $127: 232-44$.

6. Luskin MB. Restricted proliferation and migration of postnatally generated neurons derived from the forerebrain subventricular zone. Neurom $1993 ; 11$ : 173-89.

7. Lois C, Alvarez-Buylla A. Long-distance neuronal migration in the adult mammalian brain. Science 1994 ; 264 : 1145-8.

\section{ADRESSE}

A. Holley: professeurde neurosciences. Université Claude Bernard - Lyon I, laboratoire de physiologie neurosensorielle, 69622 Villeurbanne Cedex, France.

\title{
LES RÉCEPTEURS DES ODEURS ET AUTRES ATTRAITS DU MODÉLE OLFACTIF
}

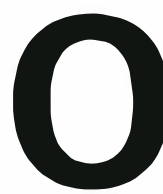
n ne trouvera plus désormais dans les revues de synthèse consacrées à l'olfaction la formule devenue rituelle : "On ne connaît pratiquement rien sur les mécanismes de la réception des odeurs ". En quatre ans, les connaissances se sont enrichies de façon décisive.

La grande famille des récepteurs olfactifs

La fin des années 1980 a vu, d'abord, l'étude des mécanismes de la transduction des signaux olfactifs déboucher sur la mise en évidence d'une première cascade enzymatique impliquant l'AMP cyclique dans le contrôle du canal ionique responsable de la dépolarisation qui conduit à la réponse électrique propagée. Vint ensuite la découverte d'une deuxième voie, celle de l'inositol triphosphate (IP3). C'est la participation avérée au processus de transduction d'une protéine liée au GTP, la protéine $G_{\text {oll }}$, qui fournit à Linda Buck et Richard Axel [1] le fil conducteur de l'élégante stratégie qui allait les mener à la découverte des récepteurs olfactifs. Les récepteurs qu'ils recherchaient devaient, en effet, appartenir à la superfamille des récepteurs couplés à des protéines $G$ et donc partager des caractéristiques structurales avec ces autres récepteurs, notamment posséder sept domaines hydrophobes transmembranaires. On trouve ainsi, au point de départ de leur expérimentation, l'utilisation d'amorces dégénérées d'oligonucléotides correspondant aux deuxième et septième régions transmembranaires de récepteurs connus.

Marc Parmentier et ses collègues, qui ont très tôt contribué à la connaissance des récepteurs olfactifs en utilisant les méthodes de la génétique moléculaire, dressent dans ce numéro (p. 1083) un tableau impressionnant des acquisitions les plus récentes. La classe des récepteurs couplés aux protéines $\mathrm{G}$ devenant l'une des plus étudiées en biologie, l'appartenance des récepteurs olfactifs à cette classe les fait bénéficier, potentiellement, d'un grand nombre de connaissances obtenues antérieurement sur d'autres membres de la classe. Il est particulièrement satisfaisant pour les biologistes de voir le domaine de la réception olf active, malgré ses propriétés singulières, suivre des principes de construction et de fonctionnement analogues à ceux qui régissent d'autres secteurs de la communication cellulaire. C'est une belle confirmation de l'unité du vivant.

Faut-il voir une autre manifestation de cette unité dans l'expression, par les cellules germinales mâles, de quelques uns des membres de la superfamille des récepteurs olfactifs? Pierre Vanderhaegen et al. exposent (p. 1083) les arguments qui plaident pour une implication de ces récepteurs dans le chimiotactisme et la mobilité du spermatozoïde.

Si grands qu'aient été les progrès accomplis, un fort contraste subsiste entre la profusion des données obtenues sur la nature des récepteurs et le peu d'indications encore disponibles sur leur fonction proprement olfactive. L'annonce de la grande diversité des récepteurs olf actifs, les interrogations sur leur degré de sélectivité, sur l'unicité ou la pluralité de leur expression dans les neurones sensoriels individuels, alimentent la réflexion des neurophysiologistes. André Holley et Gilles Sicard (p. 1091) s'efforcent d'apprécier le retentissement des découvertes récentes sur la conception du traitement de l'information sensorielle. 
La mobilisation de nombreuses équipes sur un problème central pour la compréhension du fonctionnement de l'odorat est un phénomène récent. On tenait le faible investissement de la communauté scientifique pour la conséquence du peu de considération accordée à un sens réputé en marge du processus de l'hominisation. L'évolution des primates a privilégié la vision au détriment de l'olfaction, si importante pour les autres mammifères, et on en conclut assez généralement qu'avec son statut de "microsmate" l'Homme ne tire de l'usage de son odorat que quelques maigres bénéfices dont il peut aisément se passer. Cette conclusion est largement démentie par ceux qui, à l'issue d'une rhinite ou d'un traumatisme, voient soudain leur odorat disparaître (anosmie), ou pis, leur adresser des messages ininterprétables (cacosmie). Pourtant, avec ses performances exceptionnelles en sensibilité, en étendue de spectre et en pouvoir de discrimination, le système olfactif avait de quoi susciter la curiosité des biologistes. Grâce à une conjoncture scientifique favorable, l'attrait du modèle olf actif joue désormais pleinement. Il pourrait, sous peu, s'étendre à d'autres particularités étonnantes de ce même système.

\section{Une neurogenèse ininterrompue}

Il est, en effet, une autre propriété des cellules olfactives qui mérite toute l'attention des neurobiologistes : la neurogenèse à l'âge adulte. Les neurones qui portent les récepteurs des odeurs sont remplacés lorsqu'ils dégénèrent à la suite de la section de leur axone ou de l'ablation de la structure qu'ils innervent, le bulbe olfactif. Ces neurorécepteurs, privés de cible, subissent une dégénérescence rétrograde massive qui présente les caractéristiques de la mort cellulaire programmée, ou apoptose, avec fragmentation de l'ADN nucléosomique et induction du proto-oncogène $c$-fos [2]. Le même type de mort cellulaire est observé dans l'organe olfactif au cours de l'embryogenèse [3]. Remarquons incidemment que, du fait du caractère massif des morts cellulaires induites, un excellent modèle d'étude de l'apoptose. Les neurorécepteurs dégénérés sont remplacés par de nouveaux neurones issus de la division de cellules germinales situées à la base de l'épithélium olfactif. On ignore si la néo-neurogenèse répond à un signal déclencheur ou à la disparition d'un signal inhibiteur permanent.

La neurogenèse au stade adulte n'est pas un phénomène exceptionnel suscité par des conditions expérimentales particulièrement sévères. Il est maintenant établi chez plusieurs espèces que des neurorécepteurs naissent continuellement, se différencient et deviennent fonctionnels bien après la naissance. Il est possible que ces neurones constituent une réserve en renouvellement constant qui pourvoit au remplacement des neurones lésés, au sein d'un neuroépithélium particulièrement exposé aux agents infectieux et aux produits toxiques.

Les questions posées par cette néoneurogenèse rejoignent celles que soulève le développement embryonnaire du système. On s'interroge, notamment, sur le déterminisme des connexions synaptiques entre les neurorécepteurs néoformés et leurs cibles dans le bulbe olf actif. Comment le motif plurineuronal d'activité qui représente chaque odeur individuelle préserve-t'il son identité en dépit du renouvellement permanent d'une partie au moins des neurorécepteurs afférents?

L'attention a été récemment attirée sur une catégorie de cellules gliales, les cellules " engainantes ", qui pourraient jouer un rôle essentiel dans le guidage des axones vers leurs cibles. Ces cellules, qui ont pour origine embryonnaire la placode olfactive, présentent des caractéristiques ultrastructurales et moléculaires qui les distinguent, à la fois de la glie du système nerveux central, et des cellules de Schwann du système nerveux périphérique. Au cours du développement, elles migrent précocement depuis l'épithélium olfactif, associées aux faisceaux d'axones de neurorécepteurs non myélinisés, qu'elles envelopperont ultérieurement par groupes de quelques dizaines. Dans les deux couches les plus périphériques du bulbe olfactif, cette macro- glie particulière continue de guider les axones et de les préserver des contacts avec les autres populations cellulaires. On suppose qu'à l'âge adulte les cellules « engainantes » permettent la réinnervation du bulbe olfactif par les axones des neurorécepteurs néoformés. Il vient d'être montré $[4,5]$ que l'injection d'une suspension purifiée de ces cellules, au niveau d'une racine dorsale de la moelle épinière chirurgicalement sectionnée, permettait aux axones en cours de régénération de pénétrer dans la moelle et d'y progresser vers des territoires qu'ils innervent normalement.

Le système olfactif fournit un autre exemple remarquable de neurogenèse postnatale, cette fois dans le système nerveux central. On sait que des cellules de la zone subépendymaire des ventricules latéraux continuent de proliférer chez l'adulte et peuvent donner naissance, in vitro, à des neurones. En utilisant plusieurs méthodes pour suivre, in vivo, le devenir des cellules de la zone de prolifération, chez le rat nouveau-né et la souris adulte, deux équipes $[6,7]$ ont découvert que le bulbe olfactif était la destination de neurones formés en grand nombre dans cette région. Une longue migration conduit les neurones, par une voie bien définie, jusqu'aux couches granulaire et glomérulaire du bulbe olfactif ipsilatéral où ils se différencient respectivement en cellules granulaires et cellules périglomérulaires, les deux principales catégories d'interneurones bulbaires.

Avec la taille exceptionnelle de la famille multigénique codant pour ses récepteurs, et ses formes périphérique et centrale de neurogenèse persistante, le système olf actif off re des champs d'investigation attrayants dans deux des principaux domaines de la recherche neurobiologique actuelle. Les mêmes propriétés et leurs interactions confèrent aussi à ce système une originalité propre à stimuler la production de nouveaux modèles du traitement de l'information sensorielle

\section{TIRÉS A PART}

A. Holley. 